\title{
Evaluation of residual submicroscopic Plasmodium falciparum parasites 3 days after initiation of treatment with artemisinin-based combination therapy
}

\author{
Richard Mwaiswelo ${ }^{1,2^{*}}$ and Bill Ngasala ${ }^{2}$
}

\begin{abstract}
Plasmodium falciparum resistance against artemisinin has not emerged in Africa; however, there are reports of the presence of polymerase chain reaction-determined residual submicroscopic parasitaemia detected on day 3 after artemisinin-based combination therapy (ACT). These residual submicroscopic parasites are thought to represent tolerant/resistant parasites against artemisinin, the fast-acting component of the combination. This review focused on residual submicroscopic parasitaemia, what it represents, and its significance on the emergence and spread of artemisinin resistance in Africa. Presence of residual submicroscopic parasitemia on day 3 after treatment initiation leaves question on whether successful treatment is attained with ACT. Thus there is a need to determine the potential public health implication of the PCR-determined residual submicroscopic parasitaemia observed on day 3 after ACT. Robust techniques, such as in vitro cultivation, should be used to evaluate if the residual submicroscopic parasites detected on day 3 after ACT are viable asexual parasites, or gametocytes, or the DNA of the dead parasites waiting to be cleared from the circulation. Such techniques would also evaluate the transmissibility of these residual parasites.
\end{abstract}

Keywords: Submicroscopic, Parasites, Day 3, Artemisinin-based combination therapy, Transmission, Resistance

\section{Background}

Artemisinin-based combination therapy (ACT) is one of the major malaria control tools that have played a significant role in the reduction of malaria incidences globally [1-3], with some areas such as Zanzibar reaching the elimination stage [4]. Plasmodium falciparum resistance against artemisinins has however, emerged in parts of Southeast Asia (SEA) threatening the long-term use of ACT [5-7]. The resistance is associated with polymorphisms at propeller domain of the Kelch 13 protein encoded by the $P$. falciparum pfk 13 (or $k 13$ for short) gene of the parasite, and is expressed phenotypically as

*Correspondence: richiemwai@yahoo.com

${ }^{1}$ Department of Microbiology, Immunology and Parasitology, Hubert Kairuki Memorial University, Dar es Salaam, Tanzania

Full list of author information is available at the end of the article a prolonged microscopy-determined parasite clearance time $[7,8]$. However, the $p f k 13$ gene mutations linked to resistance to artemisinins in SEA are not found in Africa, thus an in vivo microscopy-determined asexual parasites clearance time remains a reference method for the surveillance of resistance [9].

Resistance to anti-malarial drugs has often threatened malaria elimination efforts and historically has led to the resurgence of malaria incidences and deaths particularly in children $<5$ years of age in sub-Saharan Africa (SSA) $[10,11]$. Despite the recent case reports [12, 13], no artemisinin resistance has been confirmed in Africa [14-16]. Nevertheless, polymerase chain reaction (PCR)-determined residual submicroscopic parasitaemia has been reported on day 3 after case management using ACT in different parts of Africa [17-21]. Importantly, it is not well understood whether these residual submicroscopic 
parasites represent a viable resistant parasite population $[19,21,22]$, and what role they play in determining treatment outcomes, transmission dynamics and spread of artemisinin resistance $[17,19]$.

The World Health Organization (WHO) has approved five artemisinin-based combinations, including artemether-lumefantrine, artesunate-amodiaquine, artesunate-sulfadoxine-pyrimethamine, artesunatemefloquine and dihydroartemisinin-piperaquine [23]. Artemether-lumefantrine is the most widely used ACT medicine in Africa [24]. This review focused on the current knowledge on the prevalence and significance of day 3-PCR determined residual submicroscopic parasites following treatment with ACT on malaria transmission dynamics and spread of artemisinin resistance. Published reports on the treatment of uncomplicated $P$. falciparum malaria using artemether-lumefantrine, artesunateamodiaquine, artesunate-sulfadoxine-pyrimethamine, dihydroartemisinin-piperaquine and a new drug artesunate-pyronaridine [25], were included in the review.

\section{Parasite clearance time following ACT}

The major characteristic of ACT is its ability to rapidly clear asexual $P$. falciparum parasites [26-29]. ACT consists of fast acting artemisinin derivatives and slow-acting non-artemisinin partner drugs [26, 28, 29] (Table 1 ). The artemisinin component rapidly clears a large biomass of malaria parasites achieving parasite reduction on the order of $10^{8}$-fold reduction within 3 days, before the long-acting partner drug has cleared the residual parasitaemia [26, 30,31]. Artemisinins act on asexual parasites from early young ring stages to mature trophozoites, rapidly clearing the parasites [30]. The accelerated clearance of young ring-stage parasites prevents further maturation and sequestration of trophozoites, which is associated with the severity of the infection $[29,31]$. The rapid parasite clearance rate therefore, not only optimize the therapeutic benefits, but also protects both components of the combination and minimizes the risk of emergence and spread of resistant parasite populations [10]. ACT also act on young gametocytes, reducing gametocytes carriage and thus diminishes the transmission potential of the treated infection [29, 32]. However, parasite clearance following treatment with anti-malarial drug is influenced by various host, parasite and drug factors [33, 34].

Table 1 Artemisinin-based combination therapies used in Africa

\begin{tabular}{|c|c|c|c|c|c|}
\hline $\begin{array}{l}\text { Artemisinin-based } \\
\text { combination therapy }\end{array}$ & $\begin{array}{l}\text { Artemisinin } \\
\text { component }\end{array}$ & Partner drug & Partner drug target & $\begin{array}{l}\text { Presence of persistent } \\
\text { submicroscopic } \\
\text { parasites on day } 3 \\
\text { after treatment }\end{array}$ & $\begin{array}{l}\text { Validated marker } \\
\text { of drug resistance }\end{array}$ \\
\hline $\begin{array}{l}\text { Artemether-lumefan- } \\
\text { trine }\end{array}$ & Artemether & Lumefantrine & $\begin{array}{l}\text { Interferes with haem } \\
\text { detoxification }\end{array}$ & Yes $[17,19-21,25,86]$ & $\begin{array}{l}\text { pfmdr1 N86, 184F, D1246 } \\
\text { Amplification of pfmdr1 } \\
\text { copy number } \\
\text { pfcrt K76 } \\
\text { Haplotype CVMNK }[24,48 \text {, } \\
\text { 87, 88] }\end{array}$ \\
\hline $\begin{array}{l}\text { Artesunate-amodi- } \\
\text { aquine }\end{array}$ & Artesunate & Amodiaquine & $\begin{array}{l}\text { Interferes with haem } \\
\text { detoxification }\end{array}$ & Yes [89] & $\begin{array}{l}\text { pfmdr1 86Y, Y184, 1246Y } \\
\text { pfcrt 76T } \\
\text { Haplotype SVMNT }[24,87]\end{array}$ \\
\hline $\begin{array}{l}\text { Artesunate-sulfadoxine- } \\
\text { pyrimethamine }\end{array}$ & Artesunate & $\begin{array}{l}\text { Sulfadoxine } \\
\text { Pyrimethamine }\end{array}$ & $\begin{array}{l}\text { Inhibits two enzymes } \\
\text { involved in folate } \\
\text { biosynthesis pathways. } \\
\text { Sulfadoxine inhibits } \\
\text { dihydropteroate } \\
\text { synthase (dhps), } \\
\text { and Pyrimethamine } \\
\text { inhibits dihydrofolate } \\
\text { reductase (dhfr) [90, } \\
\text { 91] }\end{array}$ & Yes [90] & $\begin{array}{l}\text { pfdhfr } 511,59 \mathrm{R}, 108 \mathrm{~N} \\
\text { pfdhps } 437 \mathrm{G}, 540 \mathrm{E}[24, \\
90,91]\end{array}$ \\
\hline $\begin{array}{l}\text { Dihydroartemisinin- } \\
\text { piperaquine }\end{array}$ & Dihydroartemisinin & Piperaquine & $\begin{array}{l}\text { Not well understood but } \\
\text { linked to inhibition } \\
\text { of haem degradation } \\
\text { pathways }\end{array}$ & Yes [17] & $\begin{array}{l}\text { Plasmepsin II and III ampli- } \\
\text { fication }[24,88]\end{array}$ \\
\hline $\begin{array}{l}\text { Artesunate-pyronaridine } \\
\text { (Registered and in use } \\
\text { in some countries [23]) }\end{array}$ & Artesunate & Pyronaridine & $\begin{array}{l}\text { Interferes with haem } \\
\text { detoxification }\end{array}$ & Yes [25] & pfcrt 76T [24] \\
\hline
\end{tabular}




\section{Prolonged parasite clearance time as a measure of artemisinin resistance}

Parasite clearance time is a robust measure of efficacy of anti-malarial drugs [35]. Prolonged parasite clearance time following treatment with chloroquine, sulfadoxinepyrimethamine and mefloquine was shown previously to be a first indicator of resistance against the drugs [36, 37]. Prolonged clearance time following ACT is, therefore, viewed as first evidence of reduced susceptibility of the parasites to artemisinin and possibly preceding the clinically significant resistance [5, 31, 33, 35]. The proportion of patients with parasitaemia on day 3 following ACT is a routine indicator in monitoring $P$. falciparum sensitivity to artemisinin and its derivatives [23,38], rather than the long-acting partner drug [29]. Partial artemisinin resistance can either be suspected or confirmed. Suspected resistance to artemisinin is described as an increase in parasite clearance half-life to $\geq 5 \mathrm{~h}$, as evidenced by $\geq 10 \%$ cases with microscopy-determined parasites on day 3 after initiation of treatment with an artemisininbased combination [23, 39], or high prevalence of $p f k 13$ mutants $[9,23,39]$. The confirmed artemisinin resistance occurs when there is a combination of delayed parasite clearance and $p f k 13$ resistance-validated mutations for the same patient [38]. Importantly, there is no evidence of full artemisinin resistance; nevertheless, partial artemisinin resistance may facilitate the selection for the partner drug resistance. On the other hand, the classical drug resistance is when a parasite that normally would have been cured by a treatment regimen survives, multiplies and is transmitted to a new host [33]. Interestingly, in Africa microscopy-determined parasite clearance time is rapid following ACT, with very few patients having parasitaemia on day $3[19,35,40-42]$. However, clearance slopes in infections in SSA are likely to underestimate inherent resistance of parasites due to greater host immunity in high-transmission settings [43]. Presence of residual submicroscopic parasitaemia on day 3 , therefore, provides the early warning signs of the emergence of $P$. falciparum resistance against artemisinin-based combinations in Africa (Table 1).

\section{Residual submicroscopic parasitaemia on day 3 after ACT}

The PCR-determined submicroscopic parasites are those that are below the detection limit of a standard light microscopy, but can be detected by a much more sensitive tool, the PCR [44]. Residual PCR-determined submicroscopic malaria parasites include both asexual and sexual parasites remaining days after initiation of antimalarial treatment. ACT has continued to achieve rapid microscopy-determined parasitological clearance across SSA, with a majority of treated patients being cleared of asexual parasites within $48 \mathrm{~h}$ of treatment initiation [3, 35, 45-47]. More precisely, over $90 \%$ of patients are being microscopically parasite negative by day 2 and 99\% by day 3 post-initiation of ACT [35, 46, 47]. The PCR on the other hand, has revealed the presence in SSA of $P$. falciparum genotypes that are probably more likely to survive ACT at submicroscopic level (Table 1), and probably contribute to an onward transmission and subsequent patient recrudescence [17, 19-21, 48]. Studies in Angola [20], Kenya [17], Tanzania [18, 21], and Uganda [19] have reported substantial proportions of individuals treated with artemisinin-based combination, with residual submicroscopic parasitaemia between days 3 and 7 . Whereas a study in Angola has reported an increase, over time, in the proportion of patients with residual submicroscopic parasitaemia on day 3 [20], a study in Tanzania reported an increase followed by a decline in the proportion of patients with residual parasitaemia across years of surveillance [21]. Several submicroscopic parasite subpopulations have also been observed in Tanzania with no known pfk-13 resistance-associated mutations, however, they clear as slowly as parasites in Cambodia, which are labeled drug resistant and do harbour the mutations [43]. Interestingly, these parasites in Tanzania with similar prolonged clearance as those in Cambodia were from a study conducted in 2006 at a time when artemetherlumefantrine was not yet adopted as first-line treatment in Tanzania, and thus were probably naive of the drug $[21,43]$. Nevertheless, after nearly two decades of a widescale use of ACT in Africa, the combination therapy has remained efficacious despite the presence of residual submicroscopic parasitaemia on day $3[3,16,17,19-21]$. The questions remaining to be answered are, therefore, what are the factors other than parasite tolerance/resistance that may determine the presence of residual submicroscopic parasitaemia after ACT and whether residual PCR-determined submicroscopic parasitaemia represents viable asexual parasites.

\section{Factors associated with the presence of residual submicroscopic parasitaemia}

Apart from drug susceptibility, several factors affect the clearance of microscopy-determined parasitaemia following treatment with anti-malarial drugs leading to a large variation in clearance rates of sensitive and resistant parasite strains [9]. Parasite clearance as a predictive value for artemisinin resistance is influenced by factors including pre-treatment parasitaemia, parasite strains, parasite developmental stage, host immunity, partner drug efficacy, sample size, quality of microscopy, and the pharmacokinetic/pharmacodynamics profile of the different artemisinin derivatives and the partner drugs $[9$, $34,35]$. For instance, following anti-malarial treatment 
individuals with low pre-treatment parasitaemia clear parasitaemia more rapidly than those with higher parasitaemia. On the other hand, the burst of mature schizonts releases thousands of merozoites into the circulation increasing parasitaemia level in the peripheral blood [34]. Furthermore, sequestration of trophozoites to the inner vessels starting at around $18 \mathrm{~h}$ of the 48 -h asexual life cycle leads to a sudden decline in the level of parasitaemia in the peripheral blood.

Likewise, the same factors that affect the clearance rate of microscopy-determined parasitaemia have also shown to influence the presence of day 3 residual submicroscopic parasitaemia. Studies in Kenya and Tanzania have shown the association of the presence of residual submicroscopic parasitaemia on day 3 after initiation of ACT with higher pre-treatment microscopy-determined asexual parasitaemia, anaemia, age $<5$ years, and fever at baseline $[17,21]$. Of the factors, host immunity plays a critical role in the clearance of parasites, including resistant $P$. falciparum infections. In areas of intense transmission, acquired immunity develops at a relatively young age, and is a key determinant of the anti-malarial therapeutic response [35]. Partially immune individuals may, therefore, experience some response to drug treatment even if they are infected by drug-resistant parasites. For instance, a study in Mali has shown older children to be able to more effectively clear resistant parasites than younger children [49]. Similarly, following anti-malarial treatment, immunity is the primary determinant of clearance rate [50]. However, other studies have found no association between baseline characteristics and the presence of residual submicroscopic parasitaemia on day 3 [19].

The pharmacodynamics and pharmacokinetics of the drugs may also play a significant role in the clearance rate of malaria parasites. The fast acting component artemisinin, has a half-life of 1-2 h, clearing rapidly from the body $[26,28]$. Thus, a 3-day ACT regimen provides anti-malarial activity for two asexual parasite cycles and results in a reduction of parasitaemia in the infected individual by a factor of about 100 million parasites, but this still leaves up to 100,000 parasites for the long-acting partner drug to remove, variably assisted by the immune response [29]. Of note, the day 3 residual submicroscopic parasites are assessed at the time when the fast-acting artemisinin derivatives have already been cleared from the body; therefore, the long-acting partner drug is virtually acting alone. The day 3 PCR-determined residual parasites might therefore, be representing a remaining fraction of parasitaemia to be cleared by the long-acting partner drug after the short-acting artemisinin component has already been cleared. The observation is substantiated by the presence of residual parasitaemia in a study conducted in 2006 in Bagamoyo, Tanzania, using artemether-lumefantrine, at a time when the combination therapy was not a first-line treatment policy in the country $[18,21,43]$. At that time most of the parasites were carrying $P$. falciparum multidrug resistance-1 (mdr1) 86-Tyrosine $(\mathrm{Y})$ and $P$. falciparum chloroquine resistance transporter $(c r t)$ 76-Threonine $(\mathrm{T})$ alleles, but were known to be sensitive to artemether-lumefantrine [21]. Malaria prevalence at the time was also high in the study area, therefore, the partial immunity against malaria could be acquired at a very young age, and the study was conducted in children aged 6 months to 10 years [18]. The nearly equal proportions of submicroscopic parasitaemia on day 3 between children aged $<5$ years and the older children in the Bagamoyo study [21], probably indicates the influence of other factors for the presence of residual parasitaemia on day 3 , including selective immunity whereby some parasite populations are not affected by both the acquired immunity and drug effect [43]. Nonetheless, even though the above-mentioned factors are found to influence the presence of submicroscopic parasitaemia on day 3, more studies are needed to evaluate the viability of the detected residual asexual parasites.

\section{Efficacy of ACT in relation to day 3 residual submicroscopic parasitaemia}

Failure to rapidly clear the asexual parasite biomass by the fast-acting artemisinin component of the combination is thought to cause more parasites to be exposed to the long-acting partner drug alone increasing the risk of selection to the partner drug [38]. Prolonged parasite clearance increases the chances of more resistant asexual parasites to differentiate into gametocytes and then be transmitted after mosquito bite. Despite the presence of substantial proportion of submicroscopic parasitaemia on day 3 after $\mathrm{ACT}$, in Africa the various combination therapies have remained highly efficacious $[3,16,51]$, with the PCR-corrected efficacy reported to be above $90 \%$ in the surveyed areas $[17,19-21,47,52]$. Similar better treatment outcomes are reported in Myanmar and Vietnam despite the presence of prolonged clearance half-life of more than $5 \mathrm{~h}, \geq 10 \%$ of ACT-treated patients with microscopic-determined asexual parasites, selection of $p f k 13$ [53, 54], and residual submicroscopic parasitaemia twice as much as those with microscopy-determined parasitaemia [53]. It is, therefore, clear that delayed parasite clearance does not necessarily lead to treatment failure [38]. However, in Kenya the PCR-detected submicroscopic parasitaemia on day 3 was statistically significantly associated with PCR-adjusted recrudescence [17], but the significant association occurred after the standard WHO guideline for differentiation of recrudescence from new infection was modified [55]. Importantly, 
treatment failure rates following ACT outside the Great Mekong Sub-region occurs in the absence of artemisinin resistance, and are mainly due to partner drug resistance [56], although a cross-resistance cannot be ruled out. Treatment failure rate equal to or above $10 \%$ should prompt a change in the national anti-malarial treatment policy [38].

\section{Selection of markers of drug resistance in residual submicroscopic parasites}

Molecular markers of drug resistance are among the tools widely used for surveillance of drug sensitivity [57, 58]. Specific polymorphisms within the $P$. falciparum multidrug resistant ( $p f m d r 1)$ and $P$. falciparum chloroquine resistance transporter ( $p f c r t$ ) genes are widely used as markers for surveillance of ACT resistance in Africa [59-65], where pfk13 mutations are present but their variants differ from those in SEA and their correlation with artemisinin resistance has yet to be substantiated $[14,15,35,66]$. Both $p f m d r 1$ and pfcrt resistant haplotypes are saturated in the parasite population following years of wide-scale use of ACT, and are being detected both at baseline and in residual submicroscopic parasites detected on day 3 following ACT [21] (Table 1). Five pfmdr 1 amino acid positions asparagine (N)-86, tyrosine (Y)-184, serine (S)-1034, N1042, and aspartic acid (D)1246 influence susceptibility to lumefantrine, quinine, artemisinins and mefloquine [11]. Selection of pfmdr1 haplotypes NFD (N86Y, F184Y and D1246Y) has been consistently observed after artemether-lumefantrine treatment in Africa, and is associated with decreased parasite susceptibility to the arylaminoalcohol quinolone long-acting partner drugs, including lumefantrine [59]. On the other hand, selection of haplotype YYY is linked to decreased sensitivity to 4-aminoquinoline drugs, such as chloroquine and amodiaquine [59]. Amplification of pfmdr1 copy numbers is associated with reduced susceptibility to artemisinins, lumefantrine, and mefloquine [11]. Likewise, selection of $p f c r t$ haplotypes influence susceptibility to the commonly used partner drugs in Africa, lumefantrine and amodiaquine [11, 61]. The pfcrt 76T is associated with amodiaquine tolerance [11], whereas lysine (K)-76 reduces susceptibility to lumefantrine [21, $60,61,67]$.

While some studies have indicated evidence of statistically significant within-host directional selection of pfmdr1 NFD and pfcrt CVMNK haplotypes on day 3 post-ACT [19, 48, 68], other studies have indicated the absence of statistically significant directional selection between at baseline and on day 3 [21] or at baseline and among recurrent infections [69] over time of both pfmdr1 and pfcrt haplotypes. Random fluctuation for instance, of pfcrt is observed between day 0 and day 3, from T76 to K76 or mixed infection K76T; or from K76 to mixed infection $\mathrm{K} 76 \mathrm{~T}$ or $76 \mathrm{~T}$; or from mixed infection K76T to K76 or 76T and vice versa [21]. Similarly, there has been fluctuation of $p f m d r 1$ from $86 \mathrm{Y}$ to N86 or mixed infection N86Y; or from N86 to $86 \mathrm{Y}$ or mixed infection N86Y; or from mixed infection N86Y to N86 or $86 \mathrm{Y}$ and vice versa [21]. The lack of directional selection in some study areas is partly probably due to saturation of the parasite population, particularly with $p f m d r 1$ N86 and pfcrt K76 in these areas [21, 60]. Importantly however, the pfcrt CVMNK and pfmdr1 NFD haplotypes found in African residual submicroscopic parasites have not been directly linked to microscopy-determined delayed parasite clearance time in SEA parasites [70]. On the other hand, carriage of CVMNK prior to treatment is associated with risk of parasite recurrence on days 28 or 42 for AL, whereas the presence of NFD haplotype is not associated with risk of recrudescence [48]. However, a study in Tanzania has indicated the absence of association between both baseline and on day 3 selection of pfmdr 1 and pfcrt with recurrent infection [21]. It is worth noting that the markers of drug resistance are established when drug resistance has already developed [58].

No artemisinin resistance has been detected in Africa, and there is only partial artemisinin resistance in SEA. This might explain the lack of association between markers of drug resistance and treatment outcomes. Adequate clinical and parasitological response is observed in SEA after ACT despite the presence of parasites with $p f k 13$ mutations [53, 54]. Therefore, while molecular markers of drug resistance are very important for the surveillance of anti-malarial resistance, it should be understood that they are not often predictive of clinical resistance [31]. However, the potential to detect emerging trends of drug resistance before outright clinical failure makes molecular markers useful tools [31].

\section{Viability of residual submicroscopic asexual parasites}

The asexual parasite is the only stage of the malaria parasite responsible for pathogenesis and treatment failure. The asexual parasites can also under certain circumstances differentiate into a transmissible gametocyte stage. After ACT, a fraction of patients may continue to harbour viable microscopy-determined parasites that persist as chronic infections [19]. These tolerant or resistant asexual parasites that survives the anti-malarial drug pressure may also differentiate into gametocytes and be transmitted to the mosquito vector, where they replicate and expand the tolerant/resistant parasite population. However, the recent observation of residual submicroscopic parasitaemia 3 days after initiation of a full course treatment with an artemisinin-based drug combination 
$[19,71]$ leads to question whether these residual parasites represent viable resistant asexual parasites, which could contribute to the spread of anti-malarial drug resistance.

PCR-based detection techniques are more sensitive than microscopy [72], being able to detect as little as 2 parasites per microlitre of blood [44]. PCR uses specific primers to detect $P$. falciparum DNA targets and RNA transcripts of $18 \mathrm{~S}$ rRNA or cytochrome b [73-76], abundantly present in both asexual parasites and gametocytes [71]. Gametocytes commonly persist at low concentrations for several weeks after ACT or after ACT plus primaquine $[17,71]$. Post-treatment persistence of $18 \mathrm{~S}$ DNA or RNA in peripheral blood can thus be a consequence of the presence of both trophozoites and gametocytes. Thriemer et al., have shown that patients who are $\mathrm{qPCR}$ positive for malaria parasites at day 7 are more likely to have been carrying gametocytes before treatment and during follow-up [53]. Ring-stage RNA has also been employed to assess residual submicroscopic parasitaemia, however, the transcripts are not exclusively expressed in the ring-stage, and it is not clear whether they represent viable asexual ring-stage parasites [19]. Other ring-stage markers SBP1, REX1 and PHISTb have been used to assess submicroscopic parasitaemia on day 3 [71]. The SBP1 is most sensitive for asexual ring-stage parasites, but is also most intensely expressed in stage $\mathrm{V}$ gametocytes [71]. On the other hand, REX1 and PHISTb markers show high specificity for ring-stage trophozoites, but their specificity is compromised at gametocyte densities $>10^{6}$ gametocytes $/ \mu \mathrm{L}$, and also have lower sensitivity than SPB1 [71]. It is also not clear whether the ring-stage trophozoites detected on day 3 by these markers represent viable malaria parasites or whether mRNA transcripts may linger in the circulation after asexual parasites have been cleared [71].

Malaria parasite nuclear materials are removed from the circulation by the circulating and reticuloendothelial phagocytes [50, 77, 78]. ACT also activate the spleen to remove damaged and dead parasites from circulating infected erythrocytes through pitting, therefore, facilitating the rapid clearance of asexual parasites [30, 77-79]. Thus, DNA derived from dead parasites circulates for less than $48 \mathrm{~h}[71,73]$; and it is thought that non-viable cells are unlikely to give PCR positive signals, therefore, PCR analysis of malaria-infected blood accurately reflects the presence of live parasites [73]. Conversely, live but drug-damaged parasites that would be unable to initiate an infection when re-inoculated may also contribute to positive PCR signals [50, 71]. It is, therefore, still unclear whether the observed residual PCR-determined submicroscopic parasitaemia represents viable resistant asexual parasite strains, or are drug-damaged parasites waiting to be cleared, or are parasites remaining after the clearance of the rapid acting artemisinins and will be cleared by the long-acting partner drug.

\section{Transmission potential of residual submicroscopic parasitaemia}

Mature stage $\mathrm{V}$ gametocytes, a form of malaria parasite that develops from asexual parasites, are the only stage responsible for transmission of the infection from human to a mosquito vector [80]. Immature stage (I-IV) gametocytes are sequestering away from the peripheral circulation in a manner similar to mature asexual stage parasites [81]. Artemisinins acts against young gametocytes, significantly reducing gametocytes carriage [31, 32, 82-84], but are not potent against mature stage $\mathrm{V} P$. falciparum gametocytes. The rapid asexual parasite clearance rate exerted by artemisinins also reduces the number and window of asexual parasites to differentiate into gametocytes, therefore, reducing transmission [29, 30, 32, 82]. On the other hand, anti-malarial drug resistance is associated with increased treatment failure and gametocytes carriage. Residual submicroscopic parasitaemia are also thought to be associated with longer duration of gametocytes carriage, higher likelihood of infecting mosquitoes, a higher parasite burden in the mosquito [17], and thus further risk of infecting human hosts [48]. Mosquito membrane feeding experiments have also shown that patients with residual parasitaemia on day 3 are more likely to infect mosquito on the day of experiment (day 7) than those who have cleared asexual parasites by day 3 [17]. However, it is not known whether the PCR-determined parasites on day 3 represent viable asexual parasites that would later differentiated into gametocytes and are mature enough to be transmitted to mosquito on day 7 , the day of transmission experiment. The development and maturation of gametocytes from stage $\mathrm{I}$ to $\mathrm{V}$ requires 10-12 days [81, 85], therefore, it is almost impossible for the asexual parasites detected on day 3 to differentiate into gametocytes and be ready for transmission within 4 days. Likewise, it is not clear whether viable drug-damaged asexual parasites can differentiate into gametocytes. This does also not rule out that the positive transmissions occurring on the day of experiment are due to submicroscopic gametocytes which were present in surveyed individuals prior to the initiation of ACT. It should be emphasized that the detected PCR signals based on asexual parasites' $18 \mathrm{~s}$ rRNA are also present in gametocytes [71].

\section{Conclusion}

Presence of residual submicroscopic parasitemia on day 3 after treatment initiation leaves question on whether successful treatment is attained with ACT. Thus there is a need to determine the potential public health implication 
of the PCR-determined residual submicroscopic parasitaemia observed on day 3 after ACT. Robust techniques, such as in vitro cultivation, should be used to evaluate if the residual submicroscopic parasites detected on day 3 after ACT are viable asexual parasites, or gametocytes, or the DNA of the dead parasites waiting to be cleared from the circulation. Such techniques would also evaluate the transmissibility of these residual parasites.

\begin{abstract}
Abbreviations
ACT: Artemisinin-based combination therapy; DNA: Deoxyribonucleic acid; MeSH: Medical subject headings; mRNA: Messenger ribonucleic acid; pfcrt: Plasmodium falciparum chloroquine resistance transporter; pfmdr: Plasmodium falciparum multidrug resistance; PCR: Polymerase chain reaction; qPCR: Quantitative polymerase chain reaction; RNA: Ribonucleic acid; SEA: Southeast Asia; SSA: sub-Saharan Africa; WHO: World Health Organization.
\end{abstract}

\section{Acknowledgements}

We extend our gratitude to all authors and researchers for which through their works we have been able to produce this manuscript. We are also grateful of the support from Dr. Mwaka Kakolwa.

\section{Authors' contributions}

RM conceived the idea and wrote the manuscript; BN reviewed and edited the manuscript. All authors read and approved the final manuscript.

\section{Funding}

None.

\section{Availability of data and materials}

Not applicable.

Ethics approval and consent to participate

Not applicable.

\section{Consent for publication}

Not applicable.

\section{Competing interests}

The authors declare that they do not have any competing interest.

\section{Author details}

${ }^{1}$ Department of Microbiology, Immunology and Parasitology, Hubert Kairuki Memorial University, Dar es Salaam, Tanzania. ${ }^{2}$ Department of Parasitology and Medical Entomology, Muhimbili University of Health and Allied Sciences, Dar es Salaam, Tanzania.

Received: 15 October 2019 Accepted: 15 April 2020

Published online: 21 April 2020

\section{References}

1. WHO. World Malaria Report 2005. Geneva: World Health Organization; 2005.

2. WHO. World Malaria Report 2010. Geneva: World Health Organization; 2010.

3. WHO. World Malaria Report 2017. Geneva: World Health Organization; 2017.

4. Björkman A, Shakely D, Ali AS, Morris U, Mkali H, Abbas AK, et al. From high to low malaria transmission in Zanzibar-challenges and opportunities to achieve elimination. BMC Med. 2019;17:14

5. Dondorp A, Nosten F, Das D, Phyo AP, Tarning J, Lwin KM, et al. Artemisinin resistance in Plasmodium falciparum malaria. N Engl J Med. 2009;361:455-67.
6. Noedl H, Se Y, Sriwichai S, Schaecher K, Teja-Isavadharm P, Smith B, et al. Artemisinin resistance in Cambodia: a clinical trial designed to address an emerging problem in Southeast Asia. Clin Infect Dis. 2010;51:e82-9.

7. Ashley E, Dhorda M, Fairhurst R, Amaratunga C, Lim P, Suon S, et al. Spread of artemisinin resistance in Plasmodium falciparum malaria. N Engl J Med. 2014;371:411-23.

8. Ariey F, Witkowski B, Amaratunga C, Beghain J, Langlois A-C, Khim N, et al. A molecular marker of artemisinin-resistant Plasmodium falciparum malaria. Nature. 2014:505:50-5.

9. White LJ, Flegg JA, Phyo AP, Wiladpai-ngern JH, Bethell D, Plowe C, et al. Defining the in vivo phenotype of artemisinin-resistant falciparum malaria: a modelling approach. PLoS Med. 2015;12:e1001823.

10. Dondorp AM, Yeung S, White L, Nguon C, Day NPJ, Socheat D, et al. Artemisinin resistance: current status and scenarios for containment. Nat Rev Microbiol. 2010;8:272-80.

11. Petersen I, Eastman R, Lanzer M. Drug-resistant malaria: molecular mechanisms and implications for public health. Fed Eur Biochem Soc Lett. 2011;585:1551-62.

12. Ajayi NA, Ukwaja KN. Possible artemisinin-based combination therapyresistant malaria in Nigeria: a report of three cases. Rev da Soc Bras Med Trop. 2013;46:525-7.

13. Lu F, Culleton R, Zhang M, Ramaprasad A, von Seidlein L, Zhou H, et al. Emergence of indigenous artemisinin-resistant Plasmodium falciparum in Africa. N Engl J Med. 2017;376:991-3.

14. Taylor SM, Parobek CM, Deconti DK, Kayentao K, Coulibaly SO, Meshnick $\mathrm{SR}$, et al. Absence of putative artemisinin resistance mutations among Plasmodium falciparum in sub-Saharan Africa: a molecular epidemiologic study. J Infect Dis. 2015;211:680-8.

15. Kamau E, Campino S, Amenga-Etego L, Drury E, Ishengoma D, Johnson $\mathrm{K}$, et al. K13-propeller polymorphisms in Plasmodium falciparum parasites from sub-saharan Africa. J Infect Dis. 2015:211:1352-5.

16. WHO. World Malaria Report. Geneva: World Health Organization; 2015.

17. Beshir KB, Sutherland CJ, Sawa P, Drakeley CJ, Okell L, Mweresa CK, et al. Residual Plasmodium falciparum parasitemia in Kenyan children after artemisinin-combination therapy is associated with increased transmission to mosquitoes and parasite recurrence. J Infect Dis. 2013;208:2017-24.

18. Carlsson AM, Ngasala BE, Dahlström S, Membi C, Veiga IM, Rombo L, et al. Plasmodium falciparum population dynamics during the early phase of anti-malarial drug treatment in Tanzanian children with acute uncomplicated malaria. Malar J. 2011;10:380.

19. Chang H, Meibalan E, Zelin J, Daniels R, Eziefula AC, Meyer EC, et al. Persistence of Plasmodium falciparum parasitemia after artemisinin combination therapy: evidence from a randomized trial in Uganda. Sci Rep. 2016;6:26330.

20. Kiaco K, Teixeira J, Machado M, do Rosário V, Lopes D. Evaluation of artemether-lumefantrine efficacy in the treatment of uncomplicated malaria and its association with pfmdr1, pfatpase6 and k13-propeller polymorphisms in Luanda, Angola. Malar J. 2015;14:504.

21. Mwaiswelo R, Ngasala B, Jovel I, Xu W, Larson E, Malmberg M, et al. Prevalence of and risk factors associated with polymerase chain reactiondetermined Plasmodium falciparum positivity on day 3 after initiation of artemether-lumefantrine treatment for uncomplicated malaria in Bagamoyo district, Tanzania. Am J Trop Med Hyg. 2019;100:1179-86.

22. Abdul-Ghani R, Mahdy MAK, Beier JC, Basco LK. Hidden reservoir of resistant parasites: the missing link in the elimination of falciparum malaria. Infect Dis Poverty. 2017;6:12

23. WHO. Guidelines for the treatment of malaria. 3rd ed. Geneva: World Health Organization; 2015.

24. Nsanzabana C. Resistance to artemisinin combination therapies (ACTs): do not forget the partner drug! Trop Med Infect Dis. 2019;4:26.

25. Roth JM, Sawa P, Omweri G, Makio N, Osoti V, De Jong MD, et al. Molecular detection of residual parasitemia after pyronaridine-artesunate or artemether-lumefantrine treatment of uncomplicated Plasmodium falciparum malaria in Kenyan Children. Am J Trop Med Hyg. 2018;99:970-7.

26. Djimdé A, Lefèvre G. Understanding the pharmacokinetics of Coartem. Malar J. 2009;8(Suppl 1):S4.

27. Ezzet F, van Vugt M, Nosten F, Looareesuwan S, White N. Pharmacokinetics and pharmacodynamics of lumefantrine (benflumetol) in acute falciparum malaria. Antimicrob Agents Chemother. 2000;44:697-704. 
28. Ezzet F, Karbwang J. Population pharmacokinetics and therapeutic response of CGP 56697 (artemether + benflumetol) in malaria patients. Br J Clin Pharmacol. 1998;46:553-61.

29. White NJ. Qinghaosu (artemisinin): the price of success. Science. 2008;320:330-4

30. White NJ. Malaria parasite clearance. Malar J. 2017;16:88.

31. Lin JT. Drug-resistant malaria: the era of ACT. Curr Infect Dis Rep. 2011;12:165-73

32. White NJ. The role of anti-malarial drugs in eliminating malaria. Malar J. 2008;7(Suppl 1):S8.

33. Sibley $\mathrm{CH}$, Price RN. Monitoring antimalarial drug resistance: applying lessons learned from the past in a fast-moving present. Int J Parasitol Drugs Drug Resist. 2012;2:126-33.

34. White NJ. The parasite clearance curve. Malar J. 2011;10:278

35. WWARN. Clinical determinants of early parasitological response to ACTs in African patients with uncomplicated falciparum malaria: a literature review and meta-analysis of individual patient data. BMC Med. 2015;13:212.

36. Méndez F, Muñoz Á, Carrasquilla G, Jurado D, Arévalo-herrera M, Cortese $J F$, et al. Determinants of treatment response to sulfadoxine-pyrimethamine and subsequent transmission potential in falciparum malaria. Am J Epidemiol. 2002;156:230-8.

37. Price RN, Simpson JA, Nosten F, Luxemburger C, Hkirjaroen L, ter Kuile $F$, et al. Factors contributing to anemia after uncomplicated falciparum malaria. Am J Trop Med Hyg. 2001;65:614-22.

38. WHO. Artemisinin and artemisinin-based combination therapy resistance Geneva: World Health Organization; 2016.

39. WHO. Update on artemisinin resistance. Geneva: World Health Organization; 2012.

40. Yavo W, Faye B, Kuete T, Djohan V, Oga SA, Kassi RR, et al. Multicentric assessment of the efficacy and tolerability of dihydroartemisininpiperaquine compared to artemether-lumefantrine in the treatment of uncomplicated Plasmodium falciparum malaria in sub-Saharan Africa. Malar J. 2011;10:198

41. Kakolwa MA, Mahende MK, Ishengoma DS, Mandara Cl, Ngasala B, Kamugisha E, et al. Efficacy and safety of artemisinin-based combination therapy, and molecular markers for artemisinin and piperaquine resistance in mainland Tanzania. Malar J. 2018:17:369.

42. Adam I, Ibrahim Y, Gasim Gl. Efficacy and safety of artemisinin-based combination therapy for uncomplicated Plasmodium falciparum malaria in Sudan: a systematic review and meta-analysis. Malar J. 2018;17:110.

43. Mideo N, Jeffrey A, Hathaway N, Ngasala B, Saunders D, Lon C, et al. A deep-sequencing tool for partitioning clearance rates following antimalarial treatment in polyclonal infections. Evol Med Public Health. 2016;2016:e1093.

44. Bosman A, Cunningham J. WHO Evidence Review Group on malaria submicroscopic infections 2017. Geneva: World Health Organization; 2017.

45. Lopera-Mesa TM, Doumbia S, Chiang S, Zeituni AE, Konate DS, Doumbouya $\mathrm{M}$, et al. Plasmodium falciparum clearance rates in response to artesunate in Malian children with malaria: effect of acquired immunity. J Infect Dis. 2013;207:1655-63.

46. Maiga AW, Fofana B, Sagara I, Dembele D, Dara A, Traore OB, et al. No evidence of delayed parasite clearance after oral artesunate treatment of uncomplicated falciparum malaria in Mali. Am J Trop Med Hyg 2012:87:23-8.

47. Mwaiswelo R, Ngasala B, Jovel I, Aydin-Schmidt B, Gosling R, Premji $Z$, et al. Adding a single low-dose of primaquine $(0.25 \mathrm{mg} / \mathrm{kg})$ to artemether-lumefantrine did not compromise treatment outcome of uncomplicated Plasmodium falciparum malaria in Tanzania: a randomized, single-blinded clinical trial. Malar J. 2016:15:316.

48. Henriques $\mathrm{G}$, Hallett RL, Beshir KB, Gadalla NB, Johnson RE, Burrow R, et al. Directional selection at the pfmdr1, pfcrt, pfubp1, and pfap2mu loci of Plasmodium falciparum in Kenyan children treated with ACT. J Infect Dis. 2014;210:2001-8.

49. Djimdé A, Doumbo OK, Traore O, Guindo AB, Kayentao K, Diourte Y, et al. Clearance of drug-resistant parasites as a model for protective immunity in Plasmodium falciparum malaria. Am J Trop Med Hyg. 2003;69:558-63.

50. Hastings IM, Kay K, Hodel EM. How robust are malaria parasite clearance rates as indicators of drug effectiveness and resistance? Antimicrob Agents Chemother. 2015;59:6428-36.
51. WHO. World Malaria Report 2016. Geneva: World Health Organization; 2016.

52. Mwaiswelo R, Ngasala B, Gil JP, Malmberg M, Jovel I, Xu W, et al. Sustained high cure rate of artemether-lumefantrine against uncomplicated Plasmodium falciparum malaria after 8 years of its wide-scale use in Bagamoyo district, Tanzania. Am J Trop Med Hyg. 2017;97:526-32.

53. Thriemer K, Van Hong N, Rosanas-Urgell A, Phuc BQ, Ha DM, Pockele E, et al. Delayed parasite clearance after treatment with dihydroartemisinipiperaquine in Plasmodium falciparum malaria patients in central Vietnam. Antimicrob Agents Chemother. 2014;58:7049-55.

54. Tun KM, Jeeyapant A, Imwong M, Thein M, Soe S, Aung M, et al. Parasite clearance rates in upper Myanmar indicate a distinctive artemisinin resistance phenotype: a therapeutic efficacy study. Malar J. 2016;15:185.

55. WHO. Methods for surveillance of antimalarial drug efficacy. Geneva: World Health Organization; 2009.

56. WHO. Artemisinin resistance and artemisinin-based combination therapy efficacy. Geneva: World Health Organization; 2018.

57. Gadalla NB, Adam I, Elzaki SE, Bashir S, Mukhtar I, Oguike M, et al. Increased pfmdr 1 copy number and sequence polymorphisms in Plasmodium falciparum isolates from Sudanese malaria patients treated with artemether-lumefantrine. Antimicrob Agents Chemother. 2011;55:5408-11.

58. WHO. Global report on antimalarial drug efficacy and drug resistance: 2000-2010. Geneva: World Health Organization; 2010.

59. Ferreira PE, Holmgren G, Veiga MI, Uhlen P, Kaneko A, Gil JP. PfMDR1: mechanisms of transport modulation by functional polymorphisms. PLoS ONE. 2011:6:e23875.

60. Malmberg M, Ngasala B, Ferreira PE, Larsson E, Jovel I, Hjalmarsson A, et al. Temporal trends of molecular markers associated with artemetherlumefantrine tolerance/resistance in Bagamoyo district, Tanzania. Malar J. 2013;12:103.

61. Sisowath C, Petersen I, Veiga MI, Mårtensson A, Premji Z, Björkman A, et al. In vivo selection of Plasmodium falciparum parasites carrying the chloroquine-susceptible pfcrt K76 allele after treatment with artemetherlumefantrine in Africa. J Infect Dis. 2009;199:750-7.

62. Venkatesan M, Gadalla NB, Stepniewska K, Dahal P, Nsanzabana C, Moriera C, et al. Polymorphisms in Plasmodium falciparum chloroquine resistance transporter and multidrug resistance 1 genes: parasite risk factors that affect treatment outcomes for P. falciparum malaria after artemether-lumefantrine and artesunate-amodiaquine. Am J Trop Med Hyg. 2014;91:833-43

63. Baliraine FN, Rosenthal PJ. Prolonged selection of pfmdr 1 polymorphisms after treatment of falciparum malaria with artemether-lumefantrine in Uganda. J Infect Dis. 2011;204:1120-4.

64. Baraka V, Tinto H, Valea I, Fitzhenry R, Delgado-ratto C, Mbonye MK. Variants by artemether-lumefantrine and dihydroartemisinin- piperaquine in Burkina Faso. Antimicrob Agents Chemother. 2015:59:734-7.

65. Okombo J, Kamau AW, Marsh K, Sutherland CJ, Ochola-oyier LI. Temporal trends in prevalence of Plasmodium falciparum drug resistance alleles over two decades of changing antimalarial policy in coastal Kenya. Int J Parasitol Drugs Drug Resist. 2014;4:152-63.

66. Muwanguzi J, Henriques G, Sawa P, Bousema T, Sutherland CJ, Beshir KB. Lack of K13 mutations in Plasmodium falciparum persisting after artemisinin combination therapy treatment of Kenyan children. Malar J. 2016;15:36.

67. Malmberg M, Ferreira PE, Tarning J, Ursing J, Ngasala B, Björkman A, et al. Plasmodium falciparum drug resistance phenotype as assessed by patient antimalarial drug levels and its association with pfmdr 1 polymorphisms. J Infect Dis. 2013;207:842-7.

68. Asenath A, Chebon L, Mitei K, Opot B, Juma DW, Nyerere A, et al. Investigation of markers of artemisinin resistance at selected intervals during the $72 \mathrm{~h}$ period after artemisinin based combination therapy dosing in Kisumu, western Kenya. Adv Tech Biol Med. 2018;6:2

69. Baraka V, Mavoko HM, Nabasumba C, Francis F, Lutumba P, Alifrangis M, et al. Impact of treatment and re-treatment with amodiaquine on selection of Plasmodium falciparum multidrug resistance gene-1 polymorphisms in the Democratic Republic of Congo and Uganda. PLOS ONE. 2018;13:e0191922.

70. Lo E, Nguyen J, Oo W, Hemming-schroeder E, Zhou G, Yang Z. Examining Plasmodium falciparum and $P$ vivax clearance subsequent to antimalarial 
drug treatment in the Myanmar-China border area based on quantitative real-time polymerase chain reaction. BMC Infect Dis. 2016;16:154.

71. Tadesse FG, Lanke K, Nebie I, Schildkraut JA, Gonçalves BP, Tiono AB, et al. Molecular markers for sensitive detection of Plasmodium falciparum asexual stage parasites and their application in a malaria clinical trial. Am J Trop Med Hyg. 2017;97:188-98.

72. Pöschl B, Waneesorn J, Thekisoe O, Chutipongvivate S, Panagiotis K. Comparative diagnosis of malaria infections by microscopy, nested PCR, and LAMP in Northern Thailand. Am J Trop Med Hyg. 2010:83:56-60.

73. Jarra W, Snounou G. Only viable parasites are detected by PCR following clearance of rodent malarial infections by drug treatment or immune responses. Infect Immun. 1998;66:3783-7.

74. Schneider P, Wolters L, Schoone G, Schallig H, Sillekens P, Hermsen R, et al. Real-time nucleic acid sequence-based amplification is more convenient than real-time PCR for quantification of Plasmodium falciparum. J Clin Microbiol. 2005;43:402-5.

75. Snounou G, Viriyakosol S, Xin PZ, Jarra W, Pinheiro L, Rosario VE, et al. High sensitivity of detection of human malaria parasites by the use of nested polymerase chain reaction. Mol Biochem Parasitol. 1993;61:315-20.

76. Xu W, Morris U, Aydin-schmidt B, Msellem MI, Shakely D, Petzold M, et al. SYBR green real-time PCR-RFLP assay targeting the Plasmodium cytochrome $b$ gene - a highly sensitive molecular tool for malaria parasite detection and species determination. PLoS ONE. 2015;10:e0120210.

77. Angus BJ, Chotivanich K, Udomsangpetch R, White NJ. In vivo removal of malaria parasites from red blood cells without their destruction in acute falciparum malaria. Blood. 1997;90:2037-40.

78. Chotivanich K, Udomsangpetch R, Mcgready R, Proux S, Newton P, Pukrittayakamee $\mathrm{S}$, et al. Central role of the spleen in malaria parasite clearance. J Infect Dis. 2002;10400:1538-41.

79. Chotivanich K, Udomsangpetch R, Dondorp A, Williams T, Angus B, Simpson JA, et al. The mechanisms of parasite clearance after antimalarial treatment of Plasmodium falciparum malaria. J Infect Dis. 2000;182:629-33

80. Ouédraogo AL, Guelbéogo WM, Cohuet A, Morlais I, King JG, Gonçalves $\mathrm{BP}$, et al. A protocol for membrane feeding assays to determine the infectiousness of $P$. falciparum naturally infected individuals to Anopheles gambiae. Malaria World J. 2013;4:16.

81. Gardiner DL, Trenholme KR. Plasmodium falciparum gametocytes: playing hide and seek. Ann Transl Med. 2015;3:45.

82. Okell LC, Drakeley CJ, Ghani AC, Bousema T, Sutherland CJ. Reduction of transmission from malaria patients by artemisinin combination therapies: a pooled analysis of six randomized trials. Malar J. 2008;7:125.
83. Sawa P, Shekalaghe SA, Drakeley CJ, Sutherland CJ, Mweresa CK, Baidjoe AY, et al. Malaria transmission after artemether-lumefantrine and dihydroartemisinin-piperaquine: a randomized trial. J Infect Dis. 2013;207:1637-45.

84. Okell LC, Drakeley CJ, Bousema T, Whitty CJM, Ghani AC. Modelling the impact of artemisinin combination therapy and long-acting treatments on malaria transmission intensity. PLoS Med. 2008;5:e226.

85. Delves MJ, Ruecker A, Straschil U, Lelièvre J, Marques S, López-Barragán $\mathrm{MJ}$, et al. Male and female Plasmodium falciparum mature gametocytes show different responses to antimalarial drugs. Antimicrob Agents Chemother. 2013;57:3268-74.

86. Betson M, Sousa-Figueiredo JC, Atuhaire A, Arinaitwe M, Adriko M, Mwesigwa G, et al. Detection of persistent Plasmodium spp. infections in Ugandan children after artemether-lumefantrine treatment. Parasitology. 2014;141:1880-90.

87. Otienoburu SD, Suay I, Garcia S, Thomas NV, Srisutham S, Björkman A, et al. An online mapping database of molecular markers of drug resistance in Plasmodium falciparum: the ACT partner drug molecular surveyor. Malar J. 2019;18:12.

88. Ouji M, Augereau J, Paloque L, Benoit-Vical F. Plasmodium falciparum resistance to artemisinin-based combination therapies: a sword of Damocles in the path toward malaria elimination. Parasite. 2018;25:24.

89. Lee P, Ji D, Liu C, Rampao HS, Rosario VE, Lin I. Application of loop-mediated isothermal amplification for malaria diagnosis during a follow-up study in São Tomé. Malar J. 2012;11:408.

90. WHO. Defining and validating a measure of parasite resistance to sulfadoxine-pyrimethamine (SP) that would be indicative of the protective efficacy of SP for intermittent preventive treatment in infancy (SP-IPTi). Geneva: World Health Organization; 2009.

91. Okell LC, Griffin JT, Roper C. Mapping sulphadoxine-pyrimethamnine resistant Plasmodium falciparum malaria in infected humans and in parasite populations in Africa. Sci Rep. 2017;7:7389.

\section{Publisher's Note}

Springer Nature remains neutral with regard to jurisdictional claims in published maps and institutional affiliations.
Ready to submit your research? Choose BMC and benefit from:

- fast, convenient online submission

- thorough peer review by experienced researchers in your field

- rapid publication on acceptance

- support for research data, including large and complex data types

- gold Open Access which fosters wider collaboration and increased citations

- maximum visibility for your research: over $100 \mathrm{M}$ website views per year

At BMC, research is always in progress.

Learn more biomedcentral.com/submissions 